\section{Kidney Blood Pressure Research}

\title{
Is Peritoneal Dialysis a Suitable Renal Replacement Therapy Option for Polycystic Kidney Disease Patients?
}

\author{
Tao Zhang $^{\mathrm{a}}$ Yitian Dou ${ }^{\mathrm{a}} \quad$ Xuejun Wang $^{\mathrm{a}} \quad$ Jing Li ${ }^{\mathrm{b}} \quad$ Shili Cao ${ }^{\mathrm{a}}$ \\ aDepartment of Nephrology, First Teaching Hospital of Tianjin University of Traditional Chinese \\ Medicine, Tianjin, bDepartment of Nephrology, First Central Hospital of Tianjin, Tianjin, China
}

\section{Key Words}

Peritoneal dialysis - Polycystic kidney disease - Dialysis adequacy • Technique failure - PDassociated complications $\cdot$ All-cause mortality

\begin{abstract}
Background/Aims: Mounting clinical experience and evidence from scale observational studies have suggested that polycystic kidney disease (PKD) was not a contraindication for peritoneal dialysis (PD). Recent studies have reported that PD may be associated with a better prognosis in PKD than that of non-PKD patients. To solve the problem, we performed a systematic review and comprehensive meta-analysis to compare the outcomes between PKD and non-PKD patients on PD and the all-cause mortality between patients with PKD on PD and hemodialysis (HD). Methods: We conducted a systematic literature using electronic databases (PubMed, Ovid, Embase and Web of Science) to identify the studies reporting the endpoint events of PKD/non-PKD patients with PD and the all-cause mortality between patients with PKD on PD and HD, such as dialysis adequacy, technique failure, PD-related complications, the mode of RRT change, and all-cause mortality. We searched the literature published February 2018 or earlier. We used both fix-effects and random-effects models to calculate the overall effect estimate. A sensitivity analysis and subgroup analysis were performed to find the origin of heterogeneity. Results: 12 studies with a total of 17,040 patients reported the endpoint events of PKD/non-PKD patients with PD. No significant difference was observed on dialysis adequacy (Kt/V, SMD: -0.02, 95\%CI: -0.12-0.08; D: Pcr (4h), SMD: -0.10, 95\% CI: -0.26-0.06), technique failure (RR: 0.97, 95\%CI: 0.78-1.20), RRT change (RR: 0.96, 95\%CI: 0.77-1.19), total PDassociated complications (RR: 1.0, 95\%CI: 0.91-1.09) and all-cause mortality (RR: 0.40, 95\%CI: 0.33-0.47) in PKD patients, compared with non-PKD subjects undergoing PD. However, the proportion of renal transplantation in PKD patients was higher than that of non-PKD patients (RR: $2.04,95 \%$ CI: 1.88-2.20) with significant heterogeneity $\left(I^{2}=82.7 \%, P=0.000\right) .4$ studies with a total of 5,762 patients reported that the all-cause mortality did not differ between the PKD patients on PD and HD (RR: 0.87, 95\%CI: 0.72-1.06). Conclusion: Our meta-analysis found that the outcomes of given population of PKD patients on PD were at least not inferior as
\end{abstract}

Dr. Jing Li

and Prof. Shili Cao
Department of Nephrology, First Central Hospital of Tianjin, Tianjin 300192; Department of Nephrology, First Teaching Hospital of Tianjin University of Traditional Chinese Medicine, Tianjin 300112 (China) Tel. +86-22-27986573, E-Mail jadee_66@126.com; caoZT1949@163.com 


\section{Kidney Blood Pressure Research}

compared to those with other primary kidney diseases, and suggested that PKD might be not absolutely a contraindication for PD. Given the limitations of the proposed, it needs further large-scale studies to assess whether PD is a suitable RRT option for end-stage renal disease (ESRD) patients with PKD.

(C) 2018 The Author(s)

Published by S. Karger AG, Basel

\section{Introduction}

Polycystic kidney disease (PKD) constitutes the most common hereditary disease causing end-stage renal disease (ESRD) in the world that accounts for 6-10\% of all patients requiring renal replacement therapy (RRT) for each year [1-4]. It is characterized pathologically by progressive kidney volume secondary to fluid-filled cysts, resulting in reduced intraperitoneal space $[5,6]$. But other than that, really, abdominal wall hernias and diverticulitis are also more frequently reported in PKD patients [6-8]. The number of ESRD patients with PKD receiving RRT markedly increased, due to improved survival of those patients [4]. It has been available for about $10 \%$ of all PKD subjects who were dependent on RRT included hemodialysis (HD), peritoneal dialysis (PD) $[9,10]$ and, if feasible, kidney transplantation [11]. The decision of RRT modality is usually based upon several factors, mainly including patient's physique or choice, resource availability and, to some extent, physicians' advice which may be playing a much more pivotal role. Spithoven et al. [4] showed that the relative contribution to overall RRT decreased from 49.1 to $35.1 \%$ for HD, whereas that of PD decreased from 7.4 to $5.8 \%$ in PKD patients. On account of an increase in pre-emptive transplantation [2], the conversion in the initial modality of RRT over time suggested that the percentage of patients receiving HD that declined slightly was higher than that of non-PKD patients. However, PD has remained approximately constant throughout the study periods.

Traditionally, some nephrologists do not consider PD as a suitable RRT option for PKD patients $[9,11]$, due to the potential disadvantages of the method: increased prevalence of abdominal wall hernias, insufficient dialysis adequacy based on reduced abdominal space, peritonitis [12], dialysis fluid leaks [13], possibly increased mortality [1, 14] and so on $[7,15]$. In recent years, scale observational studies [16-20] suggested that PKD was not a contraindication for PD. Moreover, it has even been suggested that PD may be associated with a better prognosis in PKD than that of non-PKD patients [9, 16, 17, 19, 21-26]. Therefore, it has raised concern as to whether PD is appropriate for PKD patients. Given that inconsistent results, we systematically reviewed the current literature and performed a systematic review and comprehensive meta-analysis to evaluate whether PD is a suitable RRT option for PKD patients.

\section{Materials and Methods}

This meta-analysis was performed and reported according to the Preferred Reporting Items for Systematic Reviews and Meta-Analyses (PRISMA) guidelines [27].

\section{Search strategies}

Two reviewers (T. Z. and J. L.) systematically and independently searched the online databases of PubMed, Ovid, Embase and Web of Science with the last update as of February, 2018 to identify relevant studies. The following keywords were used: 'polycystic kidney disease' or 'PKD' or 'autosomal dominant polycystic kidney disease' or 'ADPKD' or 'autosomal recessive polycystic kidney disease' or 'ARPKD' or 'congenital polycystic kidney' or 'polycystic renal kidney' or 'polycystic disease of kidney' and 'peritoneal dialysis' or 'PD' or 'hemodialysis' or 'HD' 'dialysis' or 'renal replacement therapy' or 'RRT'. Titles and abstracts as well as the reference lists of all of the identified reports were examined independently in duplicate by 2 reviewers (T. Z. and J. L.) to include potentially relevant studies. 


\section{Kidney Blood Pressure Research}

\section{Inclusion criteria}

Studies that we identified were required to meet the following inclusion criteria: 1) the study design was either a prospective cohort study, retrospective cohort study or case-control studies. Individual case reports, editorials, and review articles were excluded; 2) compared the outcomes in PKD and non-PKD patients with PD, and reported all-cause mortality between patients with PKD on PD and HD; 3) clearly defined the endpoint events, included all-cause mortality, dialysis adequacy, technique failure, PD-related complications and the mode of RRT change; 4) reported data for at least one of the clinical outcomes of interest. We did not contact any organizations to obtain unpublished results and we did not identify any ongoing studies from our systematic review. Authors of conference abstracts were contacted for the published report. We did not apply any language restrictions.

\section{Selection of studies}

Two reviewers (T. Z. and J. L.) independently screened the abstracts or titles of the studies from the electronic search to identify all potential eligible studies. Potentially relevant reports were then retrieved as complete manuscripts and assessed for compliance with the inclusion criteria. Disagreements were discussed until a consensus on inclusion/exclusion was reached. For multiple study publications from the same patient cohort reporting on similar outcomes, we chose the study with the largest number of cases. For studies that presented different outcomes, we extracted outcomes from both publications.

\section{Data extraction}

Data were extracted onto standardized pro forma by one assessor (T. Z.) and then double-checked by two others (T. Z. and J. L.) from all selected articles using a standard data extraction form to determine eligibility for inclusion. The extracted data elements of this study included first author's last name, publication year, study design, sample size, participants' age and sex, history of diabetes mellitus (DM), duration of follow-up, end point events and PD-modality.

\section{Outcome measures}

The clinical outcomes for this meta-analysis were dialysis adequacy, technique failure, PD-related complications, the mode of RRT change, and all-cause mortality in PKD and non-PKD patients on PD. In addition, we also observed the all-cause mortality between patients with PKD on PD and HD.

\section{Quality assessment}

To limit the heterogeneity secondary to differences among study designs, the quality of each study was evaluated according to the guidelines developed by the United States Preventive Task Force [28] and the Evidence-Based Medicine Working Group [29]. A point score system was applied according to the quality of the study. The following characteristics were assessed: (1) clear description of inclusion and exclusion criteria; (2) study sample representative for mentioned population; (3) clear description of sample selection; (4) full specification of clinical and demographic variables; (5) follow-up duration more than one year; (6) reporting the loss of follow-up; (7) clear definition of PKD; (8) clear definition of outcomes and outcome assessment; (9) important confounders and prognostic factors identified. Studies were graded as poor quality if they met $<5$ criteria, fair if they met 5 to 7 criteria, and good if they met $\geq 8$ criteria.

\section{Statistical analysis}

For categorical variables, we calculated the relative risk (RR), as well as the corresponding 95\% confidence interval (CI) for the outcome variables of interest. Unless the outcomes used different scales, when the standardized mean difference (SMD) and 95\% CI were used. To evaluate the heterogeneity across studies, we used $I^{2}$ derived from the chi-square test, which describes the percentage of the variability in effect estimates resulting from heterogeneity, rather than sampling error (chance). An $I^{2}>50 \%$ indicates at least moderate statistical heterogeneity [30]. We performed fixed-effects meta-analysis unless there was appreciable heterogeneity ( $I^{2}$ value over $50 \%$ or chi-squared $P$-value less than 0.05 ), in which case we also performed a random-effects meta-analysis. We conducted fixed effects meta-analysis using the inverse variance method for pooling effect sizes, and random effects meta-analysis using the inverse variance heterogeneity method. 


\section{Kidney Blood Pressure Research}

Zhang et al.: Peritoneal Dialysis and Polycystic Kidney Disease

To explore sources of heterogeneity, we performed several sensitivity and subgroup analyses. The sensitivity analysis was also done in a random predefined manner to find the origin of heterogeneity. Subgroup analysis was performed on the study population (PKD or autosomal dominant polycystic kidney disease (ADPKD)), sample size $(<200$ or $\geq 200$ ), duration of follow-up ( $\geq 3$ years or $<3$ years), geographic area (Asian or not). Publication bias was evaluated using the funnel plot and an Egger's test. Statistical significance was defined as a 2-tailed $P$ value of 0.05 . All statistical analyses were performed using Stata 11 (Stata Corp LP, College Station, TX, USA).

\section{Results}

\section{Literature search}

Fig. 1 displays the study selection flow diagram. The literature search retrieved a total of 5, 691 studies. We identified 1735 duplicate studies, which were discarded. The remaining 3956 abstracts were screened further, and 3927 studies were excluded, because they were either unrelated, irrelevant, review articles or editorials. A total of 28 papers were classified initially as potentially relevant and the full papers retrieved. Of the 28 articles, 14 potential relevant papers were excluded for the following reasons: no associated endpoint events data $[12,13,15,31,32]$; did not compare PKD and Non-PKD patients [33-35]; not distinguish dialysis modalities, such as HD or PD [36, 37]; only epidemiological studies about ESRD due to PKD on PD [2, 4]; enrolled patients from the same cohorts [10, 38]. Finally, the remaining 14 articles $[9,16-26,39,40]$ were included in our meta-analysis.

\section{Description of studies}

12 studies with a total of 17,040 patients reported the endpoint events of PKD/nonPKD patients on PD. 4 studies with a total of 5,762 patients reported the all-cause mortality between patients with PKD on PD and HD. The main features of the studies on the adverse outcomes for all articles in Table 1. 7 studies [16-19, 21, 24, 39] were conducted in Europe, with the exception of seven $[9,20,22,23,25,26,40]$ conducted in Asia. Patients' age in PKD ranged from 35.4 to 63.6 years old, however, patients' age in non-PKD was higher than PKD, ranged from 46 to 61 years old. The proportion of history of DM in the studies ranged between $0 \%$ and $28.8 \%$, and the mean follow-up periods varied from 22.8 months to 5 years.

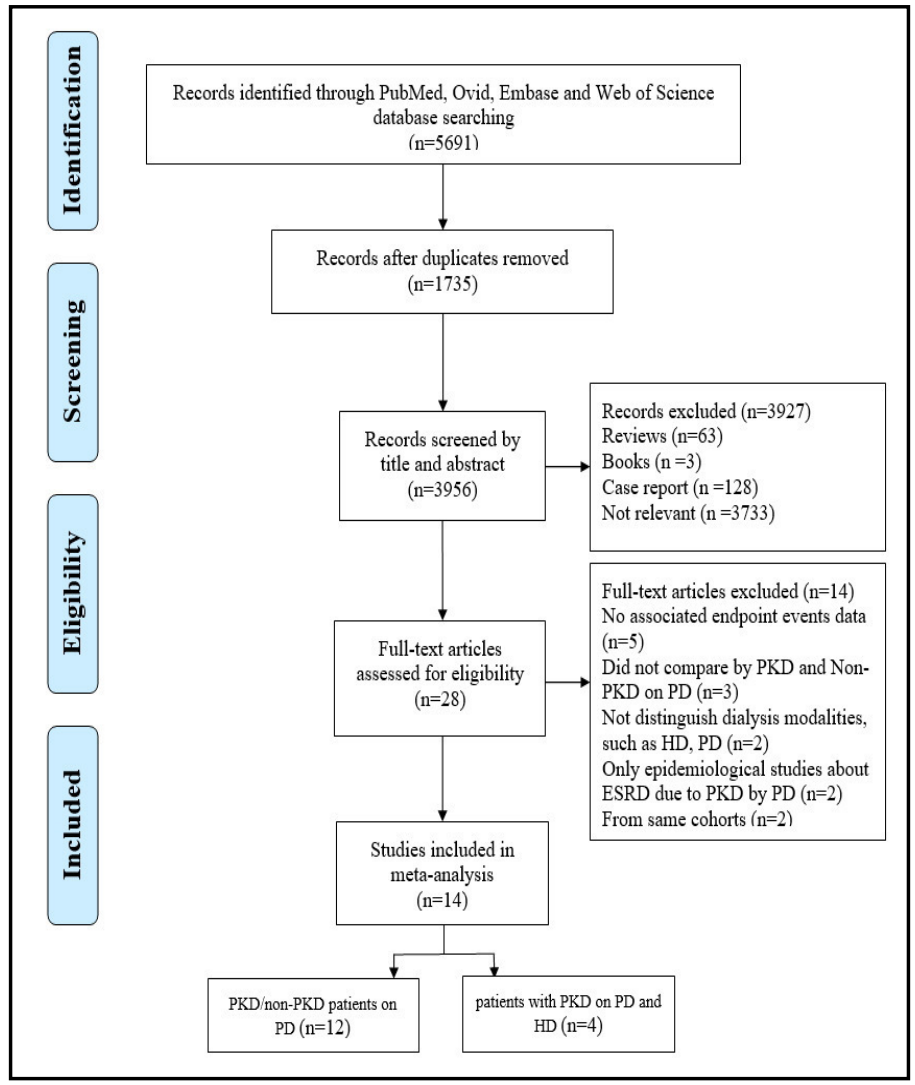

Fig. 1. Flow diagram of the selection process. PKD, polycystic kidney disease; PD, peritoneal dialysis; HD, hemodialysis; ESRD, end-stage renal disease. 


\section{Kidney \\ Bloód Pressure Research}

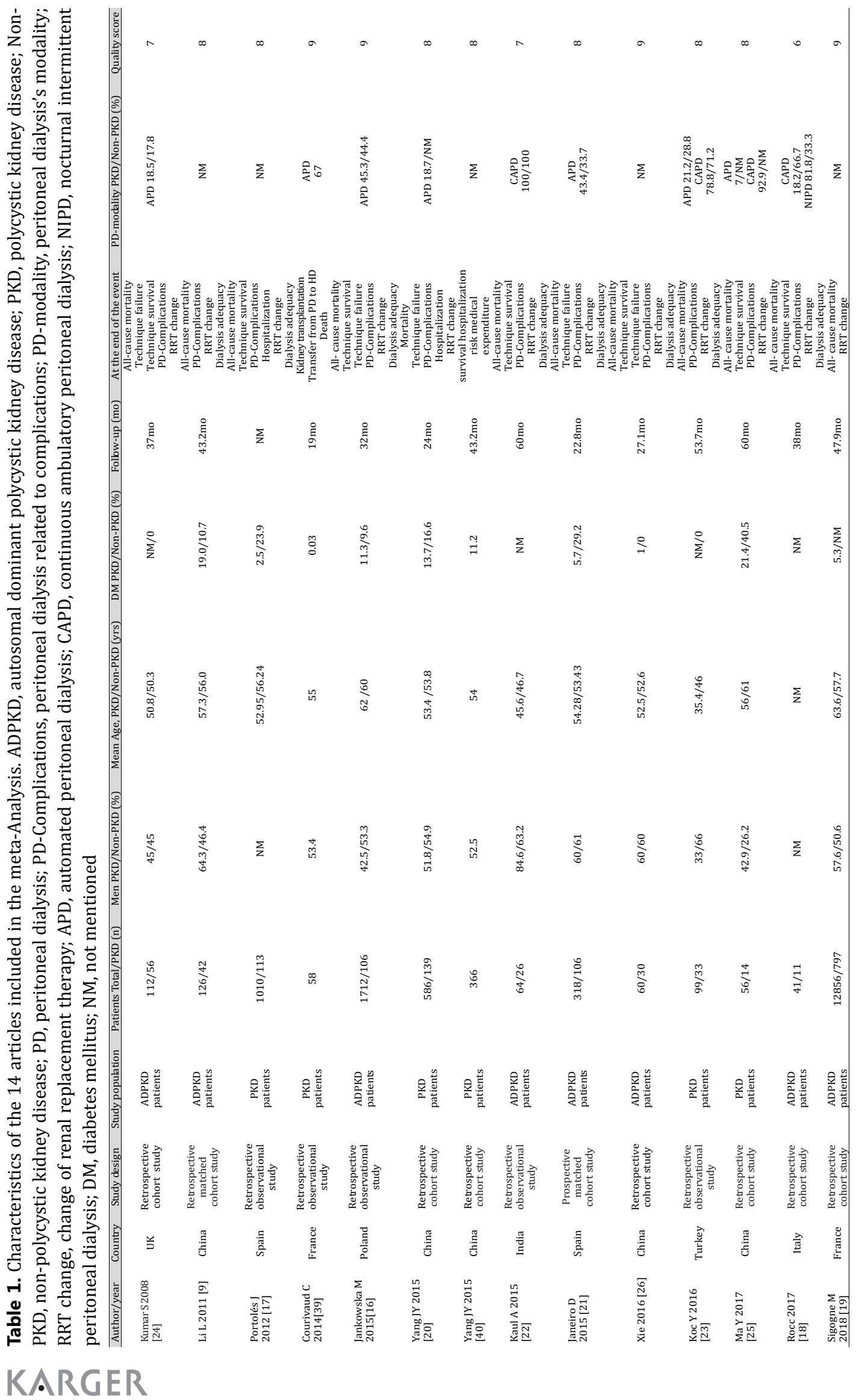




\section{Kidney Bloód Pressure Research}

The outcomes in PKD and non-PKD patients on $P D$

Dialysis adequacy. For the 9 studies $[9,16,17,21-26]$ which examined the association of $\mathrm{Kt} / \mathrm{V}$ in PKD and non-PKD patients and 4 studies [16, 22, 24, 26] examined the association of dialysate to-plasma ratios of creatinine (D: Pcr) at 4h in PKD and non-PKD patients. Whether $\mathrm{Kt} / \mathrm{V}$ or D: Pcr $(4 \mathrm{~h})$, there was no difference in terms of dialysis adequacy for each other (Kt/V, SMD: -0.02, 95\% CI: -0.12-0.08; D: Pcr (4h), SMD: -0.10, 95\%CI: -0.26-0.06). Overall, the analysis adequacy did not find a significant influence on the results (SMD: $-0.04,95 \% \mathrm{CI}$ : $-0.13-0.04)$ from all the above studies. There was no heterogeneity between the studies $\left(I^{2}\right.$ $=40 \%, P=0.067$ ) (Fig. 2).

Technique failure. Technique failure was defined as a permanent cessation of PD due to PDrelated complications. 5 studies $[16,20,21,24,26]$ examined the association of technique failure in PKD and non-PKD patients on PD. We found that there was no significantly statistical difference on technique failure in PKD and non-PKD patients on PD (RR: 0.97, 95\%CI: 0.78-1.20) without heterogeneity (Fig. 3).

$P D$-related complications. For the 9 studies $[9,16,18-20$, 22-24, 26] which examined the association of PD-related complications in PKD and nonPKD patients on PD, 7 studies [9, $16,19,20,23,24,26]$ reported peritonitis, 6 studies $[9,16,18$, $22,23,26]$ reported hernias and 5 studies [9, 16, 23, 24, 26] were leak. We found that there was no significantly statistical difference on PD-related complications in PKD and non-

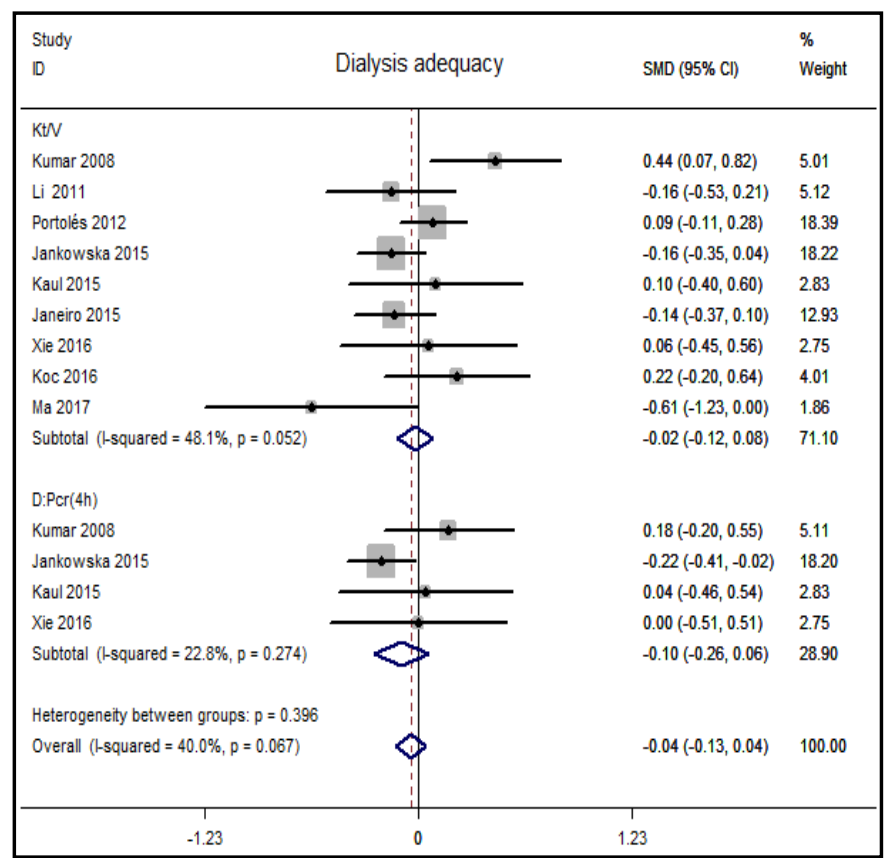

Fig. 2. Forest plot demonstrating the association of dialysis adequacy in PKD and non-PKD patients on PD. PKD, polycystic kidney disease; PD, peritoneal dialysis; SMD, standardized mean difference; $\mathrm{CI}$, confidence interval.
Fig. 3. Forest plot d e m o n s trating the association of technique failure in PKD and nonPKD patients on PD. PKD, polycystic kidney disease; PD, peritoneal dialysis; $\mathrm{RR}$, relative risk; $\mathrm{CI}$, confidence interval.

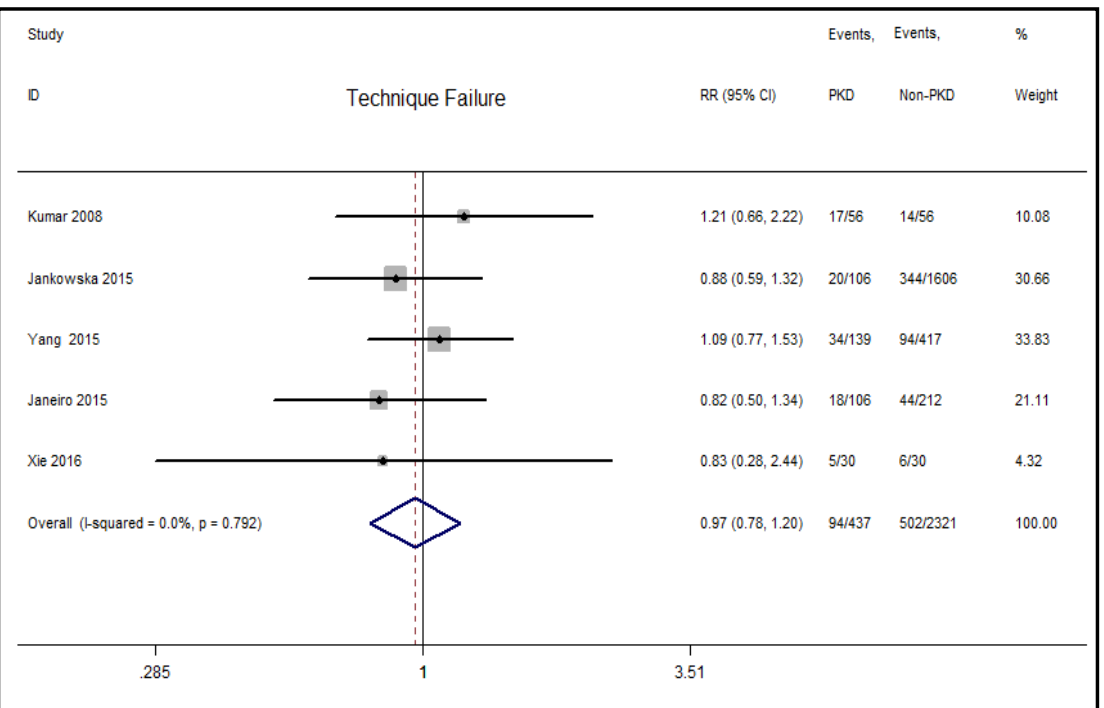




\section{Kidney Blood Pressure Research}

Fig. 4. Forest plot demonstrating the association of PDrelated complications in PKD and non-PKD patients on PD. PKD, polycystic kidney disease; PD, peritoneal dialysis; RR, relative risk; CI, confidence interval.

\begin{tabular}{|c|c|c|c|c|c|}
\hline $\begin{array}{l}\text { Study } \\
\text { ID }\end{array}$ & ed complications & $\operatorname{RR}(95 \% \mathrm{Cl})$ & $\begin{array}{l}\text { Events, } \\
\text { PKD }\end{array}$ & $\begin{array}{l}\text { Events, } \\
\text { Non-PKD }\end{array}$ & $\begin{array}{l}\% \\
\text { Weight }\end{array}$ \\
\hline \multicolumn{6}{|l|}{ Peritonitis } \\
\hline Kumar 2008 & & $0.89(0.37,2.14)$ & $8 / 56$ & 9/58 & 1.28 \\
\hline Li 2011 & & $0.33(0.04,2.88)$ & $1 / 42$ & 6/84 & 0.58 \\
\hline Jankowska 2015 & & $0.82(0.57,1.18)$ & $25 / 108$ & 484/18008 & 8.07 \\
\hline Yang 2015 & + & $1.11(0.85,1.44)$ & $49 / 139 \quad 1$ & 133/417 & 9.34 \\
\hline Xie 2016 & & $1.57(0.71,3.50)$ & $11 / 30 \quad 7$ & $7 / 30$ & 0.98 \\
\hline Koc 2018 & & $0.74(0.51,1.07)$ & $17 / 33$ & $48 / 88$ & 4.31 \\
\hline Sigogne 2018 & • & $0.93(0.84,1.03)$ & $258 / 797$ & 4184/12059 & 72.83 \\
\hline Subtotal (1-squared $=3.9 \%, p=0.397)$ & & $0.93(0.85,1.02)$ & $389 / 1203$ & 4849/14318 & 97.34 \\
\hline \multicolumn{6}{|l|}{ Hernia } \\
\hline Li 2011 & - & $4.67(1.93,11.27)$ & $14 / 42$ & 6/84 & 0.56 \\
\hline Jankowska 2015 & & $1.89(0.44,8.13)$ & $2 / 108 \quad 1$ & $16 / 1006$ & 0.28 \\
\hline Kaul 2015 & & $2.92(0.58,14.81)$ & $4 / 28$ & 2/38 & 0.23 \\
\hline Xie 2016 & & $2.00(0.40,10.11)$ & 4/30 & 2/30 & 0.28 \\
\hline Koc 2018 & & $1.50(0.38,6.31)$ & 3/33 & 4/288 & 0.37 \\
\hline Rocca 2017 & & $33.58(2.05,551.27)$ & $8 / 11$ & $0 / 30$ & 0.04 \\
\hline Subtotal $(1-$-squared $=8.7 \%, p=0.380)$ & & $3.55(2.09,6.03)$ & $33 / 248 \quad 3$ & 30/1854 & 1.76 \\
\hline \multicolumn{6}{|l|}{ Leak } \\
\hline Kumar 2008 & & $3.00(0.32,27.97)$ & 3/56 & 1/56 & 0.14 \\
\hline Li 2011 & & $5.93(0.25,142.53)$ & $1 / 42$ & 0,84 & 0.05 \\
\hline Jankowska 2015 & & $1.75(0.54,5.68)$ & 3/108 & $28 / 11008$ & 0.45 \\
\hline Xie 2018 & & $3.00(0.13,70.83)$ & 1/30 & $0 / 30$ & 0.07 \\
\hline Koc 2016 & & $4.00(0.77,20.73)$ & 4/33 & $2 / 68$ & 0.19 \\
\hline Subtotal (1-squared $=0.0 \%, p=0.910)$ & $\rightarrow$ & $2.73(1.21,6.16)$ & $12 / 287 \quad 2$ & 29/1842 & 0.90 \\
\hline Overall (1-squared $=52.5 \%, p=0.005)$ & 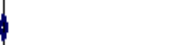 & $1.00(0.91,1.09)$ & $414 / 1718$ & 4908/18014 & 100.00 \\
\hline & & & & & \\
\hline .00181 & 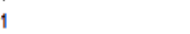 & 11 & & & \\
\hline
\end{tabular}

PKD patients on PD (RR: 1.0, 95\%CI: 0.91-1.09) with moderate heterogeneity $\left(I^{2}=52.5 \%\right.$, $P=0.005$ ) (Fig. 4).

The mode of renal replacement therapy (RRT) change

The mode of RRT change included transferred to HD and renal transplantation. For transferred to HD $[9,16-18,21,23,24,26]$, we found that there was no significantly statistical difference in PKD and non-PKD patients on PD (RR: 0.96, 95\%CI: 0.77-1.19). However, the proportion of renal transplantation $[9,16,18-26]$ in PKD patients was higher than that in non-PKD patients (RR: 2.04, 95\%CI: $1.88-2.20)$ with significant heterogeneity $\left(I^{2}=82.7 \%\right.$, $P=0.000$ ) (Fig. 5).

The all -cause mortality in PKD and non-PKD patients on PD

10 studies $[9,16,17,19,21-26]$ evaluated the all-cause mortality in PKD and non-PKD patients on PD. We found that compared to non-PKD patients with PD, all-cause mortality did not increase in PKD patients (RR: 0.40 , 95\%CI: 0.33-0.47). Heterogeneity among the studies was observed for all-cause mortality $\left(I^{2}=76.8 \%, P=0.000\right)$ (Fig. 6).

The all-cause mortality between patients with PKD on PD and HD

4 studies $[19,25,39,40]$ with a total of 5,762 patients reported the all-cause mortality between patients with PKD on PD and HD. The all-cause mortality did not differ between the PKD patients on PD and HD (RR: $0.87,95 \% \mathrm{CI}$ : $0.72-1.06)$ with no heterogeneity $\left(I^{2}=7.7 \%\right.$, $P=0.355$ ) (Fig. 7). 


\section{Kidney Blood Pressure Research}

Fig. 5. Forest plot demonstrating the association of the mode of renal replacement therapy (RRT) change in PKD and non-PKD patients on PD. PKD, polycystic kidney disease; PD, peritoneal dialysis; RRT, renal replacement therapy; $\mathrm{RR}$, relative risk; $\mathrm{CI}$, confidence interval.

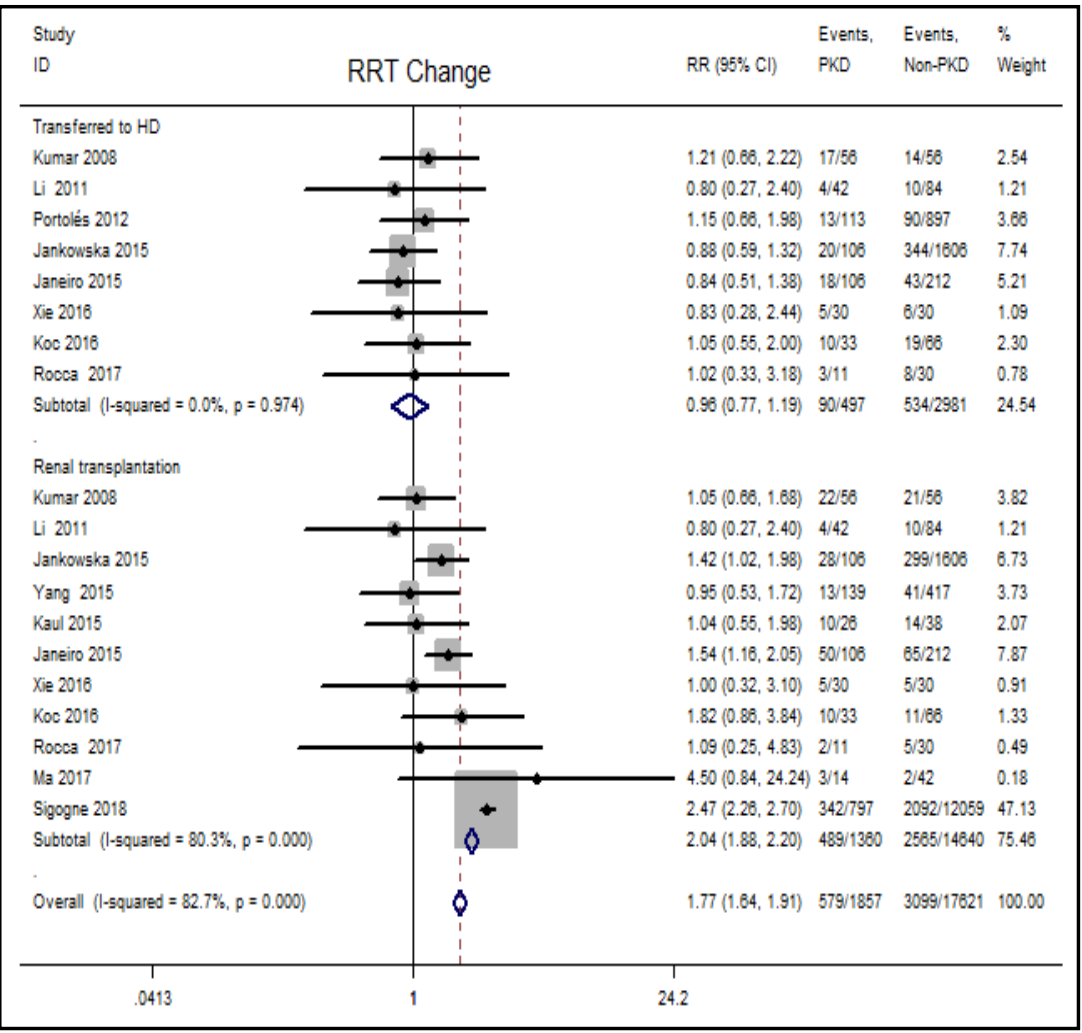

\begin{tabular}{|c|c|c|c|c|c|}
\hline Study & & & Events, & Events, & $\%$ \\
\hline ID & All cause mortality & RR (95\% Cl) & PKD & Non-PKD & Weight \\
\hline Kumar 2008 & & $1.40(0.47,4.15)$ & $7 / 56$ & $5 / 56$ & 0.89 \\
\hline Li 2011 & - & $1.13(0.71,1.81)$ & $17 / 42$ & $30 / 84$ & 3.57 \\
\hline Portolés 2012 & & $0.29(0.07,1.17)$ & $2 / 113$ & $55 / 897$ & 2.20 \\
\hline Jankowska 2015 & ل & $0.90(0.40,2.00)$ & 6/108 & $101 / 1608$ & 2.23 \\
\hline Kaul 2015 & & $1.17(0.35,3.95)$ & $4 / 26$ & $5 / 38$ & 0.73 \\
\hline Janeiro 2015 & & $0.37(0.15,0.93)$ & $5 / 106$ & $27 / 212$ & 3.21 \\
\hline Xie 2016 & & $1.50(0.27,8.34)$ & $3 / 30$ & $2 / 30$ & 0.36 \\
\hline Koc 2018 & - & $0.52(0.24,1.16)$ & $8 / 33$ & $23 / 86$ & 2.74 \\
\hline Ma 2017 & & $0.56(0.19,1.85)$ & $3 / 14$ & $16 / 42$ & 1.43 \\
\hline Sigogne 2018 & & $0.32(0.26,0.40)$ & $80 / 797$ & $3735 / 12059$ & 82.85 \\
\hline Overall (I-squared $=76.8 \%, p=0.000$ ) & & $0.40(0.33,0.47)$ & $133 / 1323$ & $3999 / 15090$ & 100.00 \\
\hline $\begin{array}{c}1 \\
071\end{array}$ & 1 & $\begin{array}{c}T \\
14\end{array}$ & & & \\
\hline
\end{tabular}

Fig. 6. Forest plot demonstrating the association of all-cause mortality in PKD and non-PKD patients on PD. PKD, polycystic kidney disease; PD, peritoneal dialysis; RR, relative risk; CI, confidence interval. 


\section{Kidney Blood Pressure Research}

\begin{tabular}{|c|c|c|c|c|c|c|}
\hline $\begin{array}{l}\text { Fig. 7. Forest plot } \\
\text { demonstrating the } \\
\text { association of all- } \\
\text { cause mortality } \\
\text { in PKD patients }\end{array}$ & $\begin{array}{l}\text { Study } \\
\text { ID }\end{array}$ & All cause mortality ( $\mathrm{PD}$ vs $\mathrm{HD}$ ) & RR $(95 \% \mathrm{Cl})$ & $\begin{array}{l}\text { Events, } \\
\text { PD }\end{array}$ & $\begin{array}{l}\text { Events, } \\
\text { HD }\end{array}$ & $\begin{array}{l}\% \\
\text { Weight }\end{array}$ \\
\hline $\begin{array}{lr}\text { on PD } & \text { and HD. } \\
\text { PKD, } & \text { polycystic } \\
\text { kidney } & \text { disease; }\end{array}$ & Courivaud 2014 & & $\rightarrow 2.36(0.62,8.95)$ & $5 / 24$ & $3 / 34$ & 1.24 \\
\hline PD, peritoneal & & & $0.71(0.46,1.10)$ & $22 / 122$ & $622 / 244$ & 20.61 \\
\hline $\begin{array}{l}\text { dialysis; } \\
\text { h e m o d i a l y s i s }\end{array}$ & & & $1.18(0.34,4.06)$ & $3 / 14$ & $6 / 33$ & 1.78 \\
\hline $\begin{array}{r}\text { R, relative risk; } \\
\text { confidence }\end{array}$ & Sigogne 2018 & & $0.88(0.71,1.10)$ & $77 / 638$ & $635 / 4653$ & 76.37 \\
\hline terval. & Overall (1-squared $=7.7 \%, p=0.355)$ & & $0.87(0.72,1.06)$ & $107 / 798$ & $706 / 4964$ & 100.00 \\
\hline & .112 & 1 & 8.95 & & & \\
\hline
\end{tabular}

Sensitivity and Table 2. Subgroup analyses of the association on the all-cause mortality subgroup analysis in PKD and non-PKD patients on PD. PKD, polycystic kidney disease; Of the 10 studies [9, $16,17,19,21-26]$ evaluated the all-cause mortality in PKD and non-PKD patients on PD, we found that compared to non-PKD patients on $\mathrm{PD}$, all-cause mortality did not increase in PKD patients on PD (RR: ADPKD, autosomal dominant polycystic kidney disease; PD, peritoneal dialysis; RR, relative risk; $\mathrm{CI}$, confidence interval

\begin{tabular}{lcccccc}
\hline \multirow{2}{*}{ Subgroup } & \multirow{2}{*}{ Study } & \multirow{2}{*}{ Number of studies } & \multicolumn{2}{c}{ Heterogeneity } & \multicolumn{2}{c}{ Meta-analysis } \\
& & & $\mathrm{I}^{2}$ & P-value & RR & $95 \% \mathrm{CI}$ \\
\hline \multirow{2}{*}{ Study population } & ADPKD & 7 & $84.2 \%$ & 0.000 & 0.39 & $0.33-0.47$ \\
Follow-up & PKD & 3 & $0.0 \%$ & 0.710 & 0.45 & $0.25-0.82$ \\
& $<3$ years & 3 & $0.0 \%$ & 0.845 & 1.02 & $0.55-1.91$ \\
Geographic area & $\geq 3$ years & 6 & $83.5 \%$ & 0.000 & 0.38 & $0.31-0.45$ \\
& Asian & 5 & $45.1 \%$ & 0.122 & 0.71 & $0.50-0.99$ \\
Sample size & Non-Asian & 5 & $74.7 \%$ & 0.003 & 0.36 & $0.29-0.43$ \\
& $<200$ & 6 & $38.6 \%$ & 0.149 & 0.76 & $0.55-1.05$ \\
& $\geq 200$ & 4 & $69.4 \%$ & 0.020 & 0.34 & $0.28-0.42$ \\
\hline
\end{tabular}

0.40 , 95\%CI: 0.33-0.47),

however, heterogeneity

among the studies was observed for all-cause mortality $\left(I^{2}=76.8 \%, P=0.000\right)$. A sensitivity analysis and subgroup analysis were performed to find the origin of heterogeneity. After removing the study by Janeiro et al. [21] which was prospective cohort study, the analysis did not find significant influences on heterogeneity across studies or overall results. Excluding the study by Koc et al. [23] with the youngest patients was also not influence on heterogeneity across studies or overall results. After excluding the study by Ma et al. [25] which had the smallest sample size and the study by Sigogne et al. [19] which had the largest sample size, no significant differences was found in the heterogeneity among the remaining 9 studies.

We also performed a predefined subgroup analyses according to study population (PKD or ADPKD), sample size ( $<200$ or $\geq 200$ ), duration of follow-up ( $\geq 3$ years or $<3$ years), geographic area (Asian or not) (Table 2). We demonstrated sample size, follow-up duration, and geographic area may be the origin of heterogeneity. In addition, our subgroup analysis showed that there was no significant influence on the risk of all-cause mortality in PKD and non-PKD patients on PD within follow-up of 3 years across 3 studies [16, 21, 26] (RR: 1.024, 95\% CI: 0.550-1.905). However, the risk of all-cause mortality in PKD patients was lower than non-PKD patients when the period of follow up was more than 3 years across 6 studies [9, 19, 22-25] (RR: 0.376, 95\% CI: 0.314-0.450). 


\section{Kidney Blood Pressure Research}

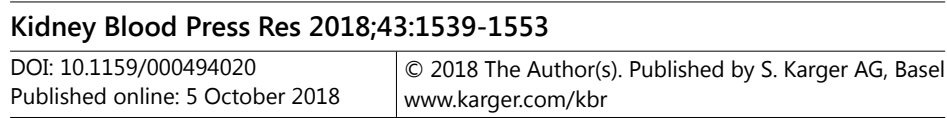

Zhang et al.: Peritoneal Dialysis and Polycystic Kidney Disease

\section{Publication bias}

The results of the funnel plot for all-cause mortality in PKD and nonPKD patients on PD were symmetrical, indicating no publication bias (Fig. 8).

\section{Discussion}

The main finding emerged from the metaanalysis is that there is no significant difference observed on dialysis adequacy, technique failure, RRT change, total PDassociated complications and all-cause mortality in PKD patients, compared

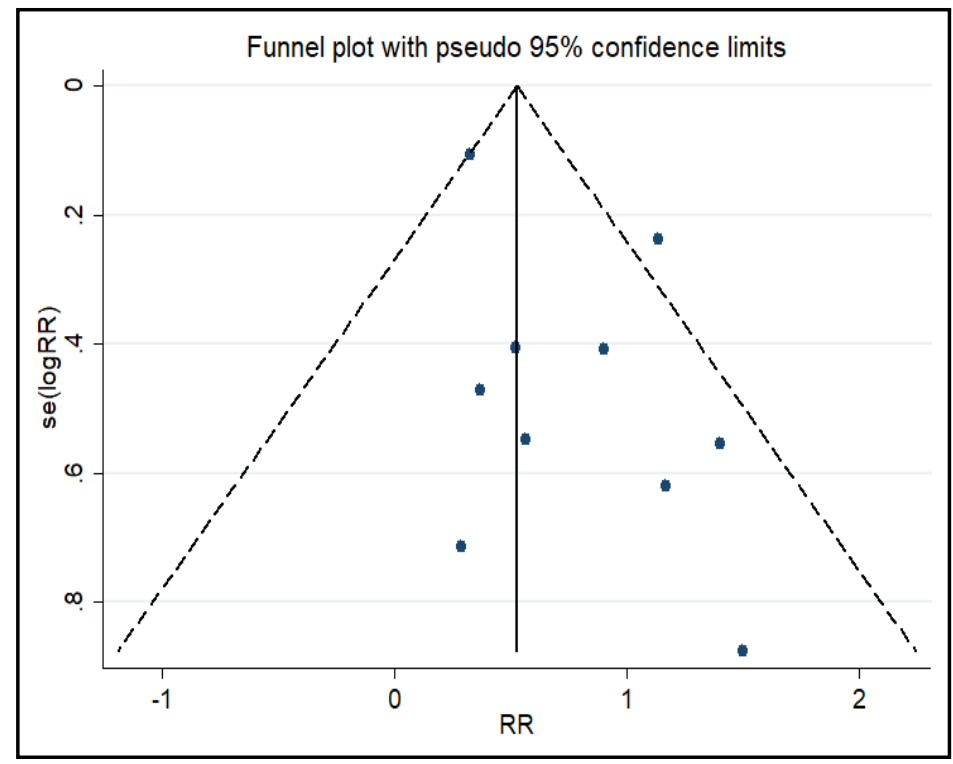

Fig. 8. Funnel plot for the association of all-cause mortality in PKD and non-PKD patients on PD. SE, standard error; RR, relative risk.

with non-PKD subjects undergoing PD. In addition, the all-cause mortality did not differ between the PKD patients on PD and HD. Due to the cognitive level, we would suggest that PKD might be not absolutely a contraindication for PD, and the outcomes of given population of PKD patients undergoing PD were at least not inferior as compared to those with other kidney diseases.

Epidemiologic studies have found that PKD was the fourth leading cause of genetic disorder related to ESRD in adults all over the world [41-43]. It is responsible for the progressive increase in volume of kidneys resulted from cyst formation gradually shrinking the size of the peritoneal cavity, and then, leading to progression of kidney failure [5, 35, 44]. Dialysis adequacy, one of the principal indicators to evaluate dialysis quality, has an important influence on the choice of dialysis modality, and even outcomes in PKD patients. As is well-known, the adequacy of PD therapy in PKD patients would be impaired by the reduced intraperitoneal volume and effective peritoneal surface area. Some studies suggested that lower dialysis efficiency was associated with reduced abdominal space secondary to the enlarged kidneys in PKD. However, our meta-analysis showed that there was no difference between the PKD group and the control group regarding dialysis adequacy. Therefore, Whether PKD patients were traditionally thought to be poorer candidates for PD therapy than those with other types of nephropathy [11] is needed to consider more factors.

To the best of our knowledge, PD-related complications might be more frequent or severe in PKD patients due to enlarged kidneys or livers which would reduce the intraperitoneal space and increase intraperitoneal pressure, including hernias, leaks, peritonitis $[9,10,12$, 24], even pre-transplant nephrectomy because of space constraints and other abdominal wall complications [45]. Taken the above potential risks into consideration, PKD often is considered as a relative contraindication to PD therapy. However, the risk of total PD-related complications, especially peritonitis including Gram-negative peritonitis, was similar in both groups in our study. Notably, it found that the incidence rate of peritonitis in PKD patients was substantially lower than that in previous reports, which was $45 \%-61 \%[7,46]$. In addition, it is of concern that a significantly increased risk for developing complications in PKD patients mostly contributed to technique failure. Several reports suggested that peritonitis may have worse outcomes and lead to early technique failure [12]. Surprisingly, our study indicated that the risk of technique failure was similar in both groups, whereas a higher rate of transfer to HD because of the specific abdominal wall causes was noted in PKD patients $[9,24,26]$. 


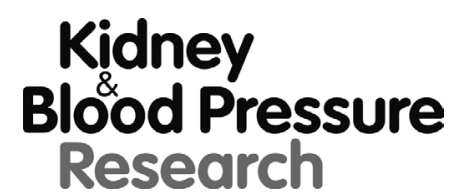

Kidney Blood Press Res 2018;43:1539-1553

DOI: 10.1159/000494020

Published online: 5 October 2018

(C) 2018 The Author(s). Published by S. Karger AG, Basel www.karger.com/kbr

Zhang et al.: Peritoneal Dialysis and Polycystic Kidney Disease

The choice of RRT modality likewise was depended on several factors, including patient's physique or choice, resource availability, public healthcare system and physicians' advice. In contrast with others [10], relying on the public health care system, patients in Spain similar to many Western countries, use a PD-first strategy. Although ESRD patients more often chose HD as an initial RRT than PD, the prevalence of PKD among patients starting HD was lower than those starting PD [21]. Interestingly, we found that there was no significant difference about conversion to HD between the PKD group and non-PKD one, but a higher incidence of renal transplantation in PKD patients than non-PKD ones. Kidney transplantation was the leading discontinuation cause of PD in some reports, particularly in PKD patients [17]. Several studies have identified younger age and lower comorbidity as factors that may explain a better kidney transplantation of PKD patients. Patients with PKD were more frequently monitored in pre-dialysis chronic kidney diseases (CKD) outpatientclinics, allowing better care and a planned access to PD in optimized conditions. They probably also had improved control of hypertension and anemia, as well as early inclusion on the transplantation waiting list. Although most probably it is a consequence of the higher proportion of transplanted patients among the ESRD subjects with PKD, compared to patients with other nephropathies, it still shows the degree of underutilization of PD treatment in the PKD population. Unfortunately, this trend may be gegenteilig observed in developed country. According to the report on the RRT in Poland, the proportion of PKD patients treated with PD constituted only $4 \%$ of dialyzed subjects in 2011 . Total health care costs were lower for PKD patients than that of control patients on dialysis. It may be gradually playing an important role on patients with PKD in ESRD that health care resource utilization and costs for those requiring dialysis were needed to be considered. Therapeutic interventions that can prevent or delay the progression in ESRD patients with PKD for increased dialysis-free life [33] were needed to be considered.

It is up to debate that whether or not the survival of PKD patients is influenced by the type of dialysis, but it is gratifying that an important conformation is being built that PD had no deleterious impact on the survival of PKD patients [19]. Recent ERA-EDTA registry study reported that mortality was lower in PKD group than non-PKD group in all RRT modalities over the last 20 years [4]. In our meta-analysis, we surprisedly found that the all-cause mortality in PKD patients was similar between PKD and non-PKD patients undergoing PD, and even a higher survival of the PKD ones. Also, the all-cause mortality did not differ between the PKD patients on PD and HD. The potential factors that PD is associated with adverse outcomes of PKD patients have not been clearly defined. Several reasons, however, have been suggested to explain why the mortality is lower than those of control group. First of all, PKD patients are younger when they deteriorate into ESRD than non-PKD patients [47], and incidence of diabetes is also lower than the control group [48]. Kumar et al. [24] found that long-term outcomes were identical in patients with PKD and in non-diabetic matched controls on PD therapy. These could explain why PKD patients had a better outcome than non-PKD groups [19,48]. Secondly, Blake et al. [49] showed that initial, as distinct from ongoing, serum albumin is an independent predictor of mortality resulted from technique failure inpatients on PD. Thirdly, cardiovascular disease aggrandized the death rate in both PKD and non-PKD patients on all RRT. Cardiovascular mortality was found to be lower in PKD patients in last period detected patients [4]. It is suggested that better risk factor management before and after initiation of RRT and improvements in quality of coronary interventions may have played a role in decreased cardiovascular mortality [5052]. Finally, they probably also had improved control of hypertension and anemia, as well as early inclusion on the transplantation waiting list. This is more relevant under an integrated RRT model promoting planned home care RRT and early transplantation [52, 53]. Taken together, these results suggest that the use of PD as RRT for PKD patients does not have a negative impact on survival. But we cannot neglect an assumption that a relative risk for the development resulted from abdominal wall hernias and peritoneal leaks in PKD patients, likely contributing to mortality. PD seems a feasible treatment option for ESRD in the course 


\section{Kidney Blood Pressure Research}

of PKD and may be always considered as an important element of an integrated therapeutic approach.

\section{Study Limitations}

The current study does have some limitations that should be considered. Firstly, all referenced studies from the meta-analysis did not have precise criteria of qualifying patients to the PD procedure, resulting in analyzed cases belonging to a biased population of patients, such as those with low total kidney volume or without a history of hernias. The above-mentioned critical factor may have a significant influence on mortality and outcomes. Secondly, given that most referenced studies from the meta-analysis pertain to retrospective cohort study, which may not randomly qualify PKD patients to PD and HD, and even presetting a fixed kidney and liver volume that would qualify patients to the PD procedure. Thirdly, heterogeneity among the studies was observed for all-cause mortality in PKD/nonPKD patients on PD, and the origin of heterogeneity may be sample size, follow-up duration, and geographic area. Fourthly, despite adjusting for multiple risk factors and prevalent diseases, it is possible that there may be residual confounding from conditions not included in the analysis, for example, population age, and diabetes. Also, the study by Sigogne et al. [19] among the included studies due to the large number of patients included $(\mathrm{n}=12,856)$ accounting for almost three quarters of the total population was the relative importance. However, our results show strong stability when analyzed with or without Sigogne's study. Finally, considering the insufficient data, we did not further to analyze other adverse outcomes, such as hospitalization.

\section{Conclusion}

Our meta-analysis suggests that no significant difference was observed on dialysis adequacy, technique failure, RRT change, total PD-associated complications and allcause mortality in PKD patients on PD, compared with non-PKD subjects. It needs to be acknowledged that for given population of PKD patients, the outcomes of PD were at least not inferior as compared to those with other primary kidney diseases. In addition, the allcause mortality did not differ between the PKD patients on PD and HD. This finding suggests that PD seems a feasible treatment option for ESRD in the given population of PKD. Given the limitations of the proposed, it needs further large-scale studies to assess whether PD is a suitable RRT option for all PKD patients.

\section{Acknowledgements}

This work was supported by Tianjin Science and Technology Program: Tianjin TCM Clinical Medicine Research Center (NO. 15ZXLCSY00020).

\section{Disclosure Statement}

The authors declare they have no conflict of interest in the publication of this article.

\section{References}

\footnotetext{
1 Abbott KC, Agodoa LY: Polycystic kidney disease at end-stage renal disease in the United States: patient
} characteristics and survival. Clin Nephrol 2002;57:208-214. 


\section{Kidney \\ Blood Pressure Research}

-2 Fernando MR, Dent H, McDonald SP, Rangan GK: Incidence and survival of end-stage kidney disease due to polycystic kidney disease in Australia and New Zealand (1963-2014). Popul Health Metr 2017;15:7.

3 Lassalle M, Ayav C, Frimat L, Jacquelinet C, Couchoud C: The essential of 2012 results from the French Renal Epidemiology and Information Network (REIN) ESRD registry. Nephrol Ther 2015;11:78-87.

4 Spithoven EM, Kramer A, Meijer E, Orskov B, Wanner C, Abad JM, Areste N, de la Torre RA, Caskey F, Couchoud C, Finne P, Heaf J, Hoitsma A, de Meester J, Pascual J, Postorino M, Ravani P, Zurriaga O, Jager KJ, Gansevoort RT, et al.: Renal replacement therapy for autosomal dominant polycystic kidney disease (ADPKD) in Europe: prevalence and survival--an analysis of data from the ERA-EDTA Registry. Nephrol Dial Transplant 2014;29:iv15-25.

5 Grantham JJ: Clinical practice. Autosomal dominant polycystic kidney disease. N Engl J Med 2008;359:1477-1485.

-6 Noel N, Rieu P: Pathophysiology, epidemiology, clinical presentation, diagnosis and treatment options for autosomal dominant polycystic kidney disease. Nephrol Ther 2015;11:213-225.

7 Del Peso G, Bajo MA, Costero 0, Hevia C, Gil F, Diaz C, Aguilera A, Selgas R: Risk factors for abdominal wall complications in peritoneal dialysis patients. Perit Dial Int 2003;23:249-254.

8 Pirson Y: Extrarenal manifestations of autosomal dominant polycystic kidney disease. Adv Chronic Kidney Dis 2010;17:173-180.

-9 Li L, Szeto CC, Kwan BC, Chow KM, Leung CB, Kam-Tao Li P: Peritoneal dialysis as the first-line renal replacement therapy in patients with autosomal dominant polycystic kidney disease. Am J Kidney Dis 2011;57:903-907.

10 Lobbedez T, Touam M, Evans D, Ryckelynck JP, Knebelman B, Verger C: Peritoneal dialysis in polycystic kidney disease patients. Report from the French peritoneal dialysis registry (RDPLF). Nephrol Dial Transplant 2011;26:2332-2339.

11 Singh S, Hariharan S: Renal replacement therapy in autosomal dominant polycystic kidney disease. Nephron 1991;57:40-44.

-12 Pandya BK, Friede T, Williams JD: A comparison of peritonitis in polycystic and non-polycystic patients on peritoneal dialysis. Perit Dial Int 2004;24:79-81.

13 De V, Scalamogna A, Scanziani R, Castelnovo C, Dozio B, Rovere G: Polycystic kidney disease and late peritoneal leakage in CAPD: are they related? Perit Dial Int 2002;22:82-84.

14 Perrone RD, Ruthazer R, Terrin NC: Survival after end-stage renal disease in autosomal dominant polycystic kidney disease: contribution of extrarenal complications to mortality. Am J Kidney Dis 2001;38:777-784.

15 Fletcher S, Turney JH, Brownjohn AM: Increased incidence of hydrothorax complicating peritoneal dialysis in patients with adult polycystic kidney disease. Nephrol Dial Transplant 1994;9:832-833.

16 Jankowska M, Chmielewski M, Lichodziejewska-Niemierko M, Jagodzinski P, Rutkowski B: Peritoneal dialysis as a treatment option in autosomal dominant polycystic kidney disease. Int Urol Nephrol 2015;47:1739-1744.

17 Portolés J, Uta S, Tato AM, Sanchez PL, Ribera M, Pena RR, Del Peso G, Ortega M, Felipe C: Polycystic Kidney Disease should not be considered as a formal contraindication for peritoneal dialysis. Nephrol Dial Transplant 2012; DOI:10.1093/gfs243.

18 Rachele Rocca A, Gnerre Musto T, Testorio M, Nunzi A, Zavatto A, Forte F, Filippini A: Peritoneal dialysis as the first-line renal replacement therapy in patients with autosomical dominant polycystic kidney disease. Nephrol Dial Transplant 2017:iii636-637.

19 Sigogne M, Kanagaratnam L, Dupont V, Couchoud C, Verger C, Maheut H, Hazzan M, Halimi JM, Barbe C, Canivet E, Petrache A, Dramé M, Rieu P, Touré F: Outcome of autosomal dominant polycystic kidney disease patients on peritoneal dialysis: a national retrospective study based on two French registries (the French Language Peritoneal Dialysis Registry and the French Renal Epidemiology and Information Network). Nephrol Dial Transplant 2018; DOI:10.1093/gfx364.

-20 Yang JY, Chen L, Chao CT, Peng YS, Chiang CK, Kao TW, Chien KL, Wu HY, Huang JW, Hung KY: Outcome comparisons between patients on peritoneal dialysis with and without polycystic kidney disease: a nationwide matched cohort study. Medicine (Baltimore) 2015;94:e2166. 


\section{Kidney \\ Blood Pressure Research}

Zhang et al.: Peritoneal Dialysis and Polycystic Kidney Disease

21 Janeiro D, Portoles J, Tato AM, Lopez-Sanchez P, Del Peso G, Rivera M, Castellano I, Fernandez-Reyes MJ, Perez-Gomez V, Ortega M, Martínez-Miguel P, Felipe C, Caparrós G, Ortiz A, Selgas R, Grupo Centro de Diálisis Peritoneal (GCDP): Peritoneal dialysis can be an option for dominant polycystic kidney disease: an observational study. Perit Dial Int 2015;35:530-536.

-22 Kaul A, Dharshan R, Bhadhuaria D, Prasad N, Gupta A, Sharma RK: Is CAPD a viable option among ADPKD with end stage renal disease population in India? Its outcomes and economics. Saudi J Kidney Dis Transpl 2015;26:906-911.

-23 Koc Y, Basturk T, Sakaci T, Atan Ucar Z, Ahbap E, Sevinc M, Sinangil A, Kara E, Ozdemir Kayalar A, Bayraktar Caglayan F, Sahutoglu T, Baris Hasbal N, Unsal A: Is peritoneal dialysis a therapeutic option for polycystic kidney disease? 15 years' experience in a single center. Nephrol Ther 2016;12:215-220.

-24 Kumar S, Fan SL, Raftery MJ, Yaqoob MM: Long term outcome of patients with autosomal dominant polycystic kidney diseases receiving peritoneal dialysis. Kidney Int 2008;74:946-951.

25 Ma Y, Wang HY, Zhou ZJ, Li Y, Yang W, Liu BY, Xia JH, Li XM, Chen LM: Effectiveness of peritoneal dialysis in treating adult end stage renal disease patients with polycystic kidney disease. Zhongguo Yi Xue Ke Xue Yuan Xue Bao 2017;39:485-491.

26 Xie XS, Xie ZT, Xiang SL, Yan XQ Zhang XH, Shou ZF, Chen JH: Peritoneal dialysis for autosomal dominant polycystic kidney disease: a retrospective study. J Zhejiang Univ Sci B 2016;17:375-381.

27 Moher D, Liberati A, Tetzlaff J, Altman DG: Preferred reporting items for systematic reviews and metaanalyses: the PRISMA statement. Int J Surg 2010;8:336-341.

28 Harris RP, Helfand M, Woolf SH, Lohr KN, Mulrow CD, Teutsch SM, Atkins D: Current methods of the US Preventive Services Task Force: a review of the process. Am J Prev Med 2001;20:21-35.

29 Levine M, Walter S, Lee H, Haines T, Holbrook A, Moyer V: Users' guides to the medical literature. IV. How to use an article about harm. Evidence-Based Medicine Working Group. JAMA 1994;271:1615-1619.

-30 Higgins JP, Thompson SG, Deeks JJ, Altman DG: Measuring inconsistency in meta-analyses. BMJ 2003;327:557-560.

-31 Hadimeri H, Johansson AC, Haraldsson B, Nyberg G: CAPD in patients with autosomal dominant polycystic kidney disease. Perit Dial Int 1998;18:429-432.

-32 Neureiter D, Frank H, Kunzendorf U, Waldherr R, Amann K: Dialysis-associated acquired cystic kidney disease imitating autosomal dominant polycystic kidney disease in a patient receiving long-term peritoneal dialysis. Nephrol Dial Transplant 2002;17:500-503.

-33 Brunelli SM, Blanchette CM, Claxton AJ, Roy D, Rossetti S, Gutierrez B: End-stage renal disease in autosomal dominant polycystic kidney disease: a comparison of dialysis-related utilization and costs with other chronic kidney diseases. Clinicoecon Outcomes Res 2015;7:65-72.

-34 Lu YA, Tu KH, Lee CC, Wu PW, Chang CJ, Tian YC, Yang CW, Chu PH: Prognostic impact of peritonitis in hemodialysis patients: a national-wide longitudinal study in Taiwan. PLoS One 2017;12:e0173710.

-35 Sharma K, Caroli A, Quach LV, Petzold K, Bozzetto M, Serra AL, Remuzzi G, Remuzzi A: Kidney volume measurement methods for clinical studies on autosomal dominant polycystic kidney disease. PLoS One 2017;12:e0178488.

-36 Lee PW, Chien CC, Yang WC, Wang JJ, Lin CC: Epidemiology and mortality in dialysis patients with and without polycystic kidney disease: a national study in Taiwan. J Nephrol 2013;26:755-762.

-37 Ritz E, Zeier M, Schneider P, Jones E: Cardiovascular mortality of patients with polycystic kidney disease on dialysis: is there a lesson to learn? Nephron 1994;66:125-128.

38 Portoles J, Janeiro D, Tato AM, Lopez P, Castellano I, Peso GD, Rivera M, Fernández-Reyes MJ, Ortega M, Miguel PMD, Caparrós G, Selgas R, Register Of Peritoneal Dialysis Center Group: Peritoneal dialysis can be an option for autosomal dominant polycystic kidney disease: a multicentre observational study. Nephrol Dial Transplant 2014;29:iii223-iii232.

39 Courivaud C, Roubiou C, Delabrousse E, Bresson-Vautrin C, Chalopin JM, Ducloux D: Polycystic kidney size and outcomes on peritoneal dialysis: comparison with haemodialysis. Clin Kidney J 2014;7:138-143.

40 Yang JY, Chen L, Chao CT, Peng YS, Chiang CK, Kao TW, Chien KL, Wu HY, Huang JW, Hung KY: Comparative Study of Outcomes among Patients with Polycystic Kidney Disease on Hemodialysis and Peritoneal Dialysis. Sci Rep 2015;5:12816. 


\section{Kidney \\ Blood Pressure Research}

Kidney Blood Press Res 2018;43:1539-1553

\begin{tabular}{l|l}
\hline DOI: $10.1159 / 000494020$ & (c) 2018 The Author(s). Published by S. Karger AG, Basel
\end{tabular}

Published online: 5 October 2018

www.karger.com $/ \mathrm{kbr}$

Zhang et al.: Peritoneal Dialysis and Polycystic Kidney Disease

41 Wakai K, Nakai S, Kikuchi K, Iseki K, Miwa N, Masakane I, Wada A, Shinzato T, Nagura Y, Akiba T: Trends in incidence of end-stage renal disease in Japan, 1983-2000: age-adjusted and age-specific rates by gender and cause. Nephrol Dial Transplant 2004;19:2044-2052.

42 Zuo L, Wang M: Current burden and probable increasing incidence of ESRD in China. Clin Nephrol 2010;74:S20-22.

-43 Collins AJ, Foley RN, Chavers B, Gilbertson D, Herzog C, Ishani A, Johansen K, Kasiske BL, Kutner N, Liu J, St Peter W, Guo H, Hu Y, Kats A, Li S, Li S, Maloney J, Roberts T, Skeans M, Snyder J, et al.: US Renal Data System 2013 Annual Data Report. Am J Kidney Dis 2014, 63:A7.

-44 Torres VE, Harris PC, Pirson Y: Autosomal dominant polycystic kidney disease. Lancet 2007;369:12871301.

45 Fuller TF, Brennan TV, Feng S, Kang SM, Stock PG, Freise CE: End stage polycystic kidney disease: indications and timing of native nephrectomy relative to kidney transplantation. J Urol 2005;174:22842288.

46 Morris-Stiff G, Coles G, Moore R, Jurewicz A, Lord R: Abdominal wall hernia in autosomal dominant polycystic kidney disease. Br J Surg 1997;84:615-617.

-47 Spithoven EM, Kramer A, Meijer E, Orskov B, Wanner C, Caskey F, Collart F, Finne P, Fogarty DG, Groothoff JW, Hoitsma A, Nogier MB, Postorino M, Ravani P, Zurriaga O, Jager KJ, Gansevoort RT; ERA-EDTA Registry; EuroCYST Consortium; WGIKD; et al.: Analysis of data from the ERA-EDTA Registry indicates that conventional treatments for chronic kidney disease do not reduce the need for renal replacement therapy in autosomal dominant polycystic kidney disease. Kidney Int 2014;86:1244-1252.

-48 Portoles J, Corchete E, Lopez-Sanchez P, Coronel F, Ocana J, Ortiz A: Patients on peritoneal dialysis with type 2 diabetes present poorer progress than non-diabetics at the expense of their cardiovascular comorbidity. Nefrologia 2009;29:336-342.

-49 Blake PG, Flowerdew G, Blake RM, Oreopoulos DG: Serum albumin in patients on continuous ambulatory peritoneal dialysis--predictors and correlations with outcomes. J Am Soc Nephrol 1993;3:1501-1507.

$>50$ Baker WL, Coleman CI, Kluger J, Reinhart KM, Talati R, Quercia R, Phung OJ, White CM: Systematic review: comparative effectiveness of angiotensin-converting enzyme inhibitors or angiotensin II-receptor blockers for ischemic heart disease. Ann Intern Med 2009;151:861-871.

51 Patch C, Charlton J, Roderick PJ, Gulliford MC: Use of antihypertensive medications and mortality of patients with autosomal dominant polycystic kidney disease: a population-based study. Am J Kidney Dis 2011;57:856-862.

52 Hou W, Lv J, Perkovic V, Yang L, Zhao N, Jardine MJ, Cass A, Zhang H, Wang H: Effect of statin therapy on cardiovascular and renal outcomes in patients with chronic kidney disease: a systematic review and metaanalysis. Eur Heart J 2013;34:1807-1817.

53 Portoles J, Moreno F, Lopez-Sanchez P, Mancha J, Gomez M, Corchete E, Del Peso G, Bajo MA, LlopezCarratala R, Fernandez-Perpen A: Peritoneal dialysis and kidney transplant. a two-way ticket in an integrated renal replacement therapy model. Nefrologia 2011;31:441-448. 